\title{
Design of Rechargeable Air Pressure Sensor Based on ZigBee Wireless Network Technology
}

\author{
Jian Chen ${ }^{1, ~ a ~}$, Keke $\mathrm{Li}^{1, \mathrm{~b}}$, Jieyang Wang ${ }^{1, \mathrm{c}}$, Junyao Tang ${ }^{1, \mathrm{~d}}$ \\ ${ }^{1}$ School of Electromechanical Engineering, Guangdong University of Technology, Guangzhou, \\ 510006, China \\ aemail: chenjian@gdut.edu.cn, ${ }^{\mathrm{b} e m a i l}: 15622782729 @ 163 . c o m,{ }^{c}$ email:jieyangw@126.com, \\ demail:yao3238181@163.com
}

Keywords: ZigBee; CC2530; air pressure sensor; intelligent charging;IOT

\begin{abstract}
We have made some improvements in the design of its new generation for the first generation of the wireless pressure sensor that has been successfully used [1][2]. The sensor is mainly applied for monitoring air pressure of airbag during the process of operating the ship. As for the design of sensor circuit, TI BQ24072 charge management chips are used to realize online charging, so sensors can work continuously and meet the needs of the construction site. And experiments show that the improved pressure sensors work stably and get the charging function.
\end{abstract}

\section{Introduction}

The technology of airbag ship launching construction is one of the independent intellectual property rights in our country, which is suitable for small and medium-sized ship launching construction technology. A large number of airbags used will lift ships, so they will move on the cluster of gasbags and then enter into water. During the whole project, the key step to finish this work successfully is to monitor the pressure of the group of airbags timely and accurately. The air pressure sensor networks of gasbag group grounded on the ZigBee wireless protocol in this project will gather air pressure sensor signal installed in every senior in the network coordinator. Then the communication between the coordinator and upper machine meet the display, storage and record of the pressure of the airbag group on the upper PC machine. Because the single section of lithium ion battery capacity of sensor nodes is limited and can't meet continuous and periodic monitoring during construction. This paper has optimized and improved the terminal node circuit, Moreover, it has further developed sensor nodes with online charge function on the basis of the achieved accomplishments, and increased the sleep mode in the software control. All of those above has improved the practicability of the system significantly.

\section{The Structure and Working Principles of the Air Pressure Monitoring System of the Gasbag Group}

\section{The air pressure sensor network of ZigBee wireless gasbag group}

The system is mainly composed of the terminal node collecting pressure, ZigBee router, ZigBee coordinator and the monitoring center from upper machines, shown in Figure 1. ZigBee wireless sensor network protocol is the wireless communication technology with a short distance, lower complexity and lower power consumption, high intelligentization, and low cost. And its standard is IEEE802.15.4, work frequency is $2.4 \mathrm{GHz}$, transmission range is $75 \mathrm{~m}$ and can reach $1 \sim 3 \mathrm{~km}$ after increasing transmitting power[3]. According to the practical need of monitoring airbags pressure group, three main functional devices are in network, such as ZigBee coordinator, ZigBee router and ZigBee terminal pressure sensor nodes. In this device, the coordinator is responsible for network establishment and management, data transfer and external network connections. While the pressure sensor terminal node is mainly used to send the collected air pressure data to the coordinator. The coordinators, routers and terminal nodes all have used TI's CC2530 chip, which is the second generation ZigBee/IEEE802.15.4 SOC chip launched by TI. The enhanced 8051 kernels that are 
standard in this field are embedded in the chips, the RF transceivers with excellent performance and the chip supports ZigBee Pro-2007 feature set [4][5]. But you have to set a good working channel and a PAN logo before the ZigBee network starting working.

\section{The Function of the ZigBee Wireless Air Pressure Sensor Terminal}

1) Realizing the acquisition and wireless transmission of the air pressure data, terminal nodes integrating Freescale MPX5700's pressure sensors, CC2530 control and RF transceiver chips, so realizing pressure data acquisition and wireless transmission.

2) Supporting the online charging of terminal nodes, integrating intelligent charging management chips and microUSB interfaces, so realizing the node online charging and improving work efficiency.

3) Controlling software sleep and awaking and prolonging battery's life.

4) Having waterproof function and meeting the requirements of outdoor environment's work.

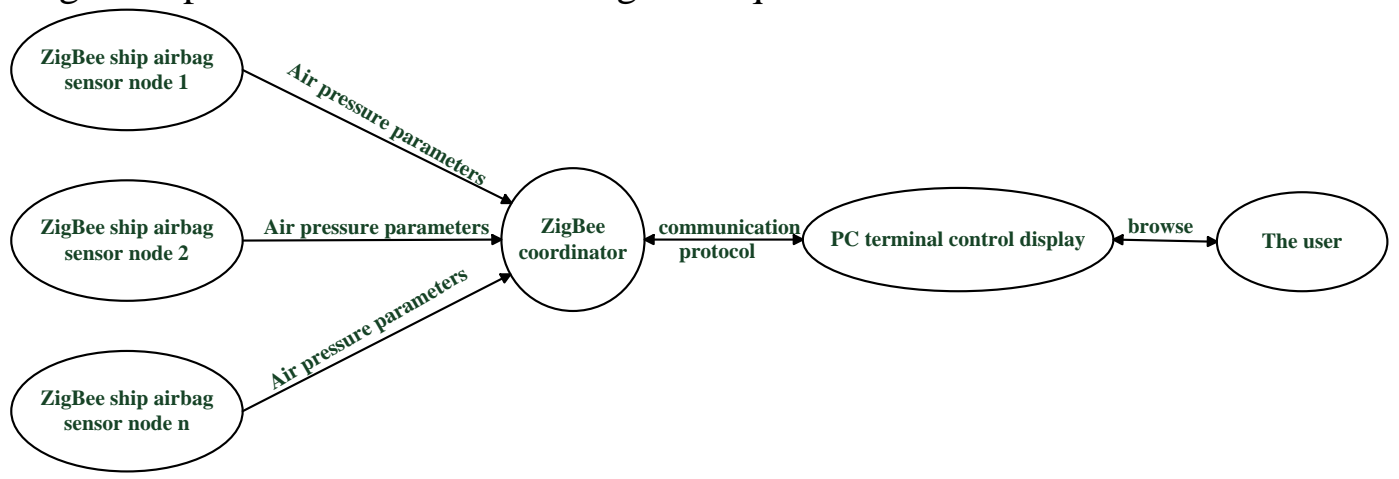

Fig.1. The monitoring system of air pressure of ZigBee gasbag group

\section{Hardware Design of ZigBee Wireless Pressure Sensor Enddevice}

The ZigBee wireless pressure sensor end device is made up of four modules, including pressure sensor, charging, wireless transmission, and the conversion circuit. The module structure is shown in Figure 2. and the unassembled object is shown in Figure 3.

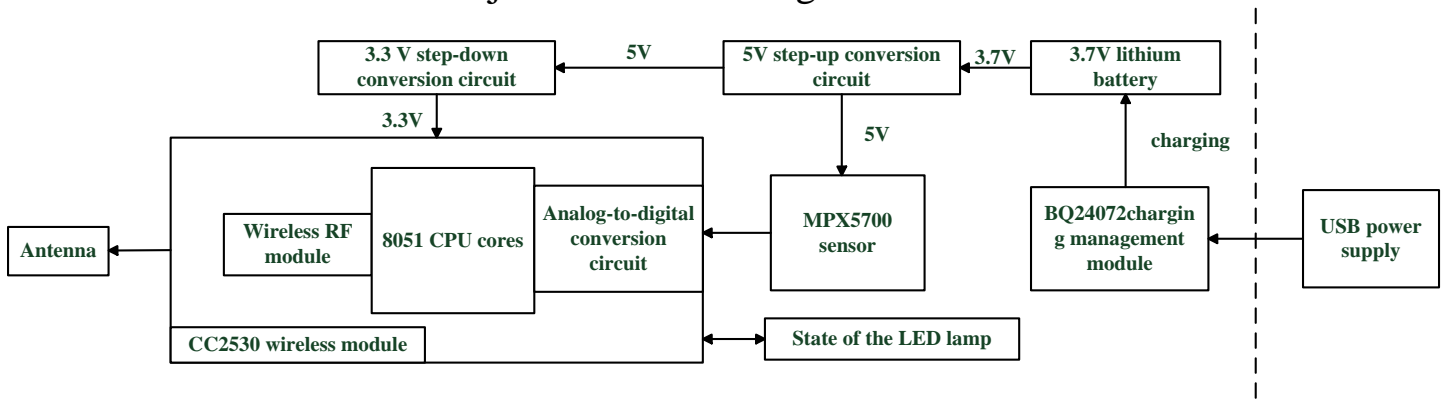

Fig.2. circuit of ZigBee wireless pressure sensor

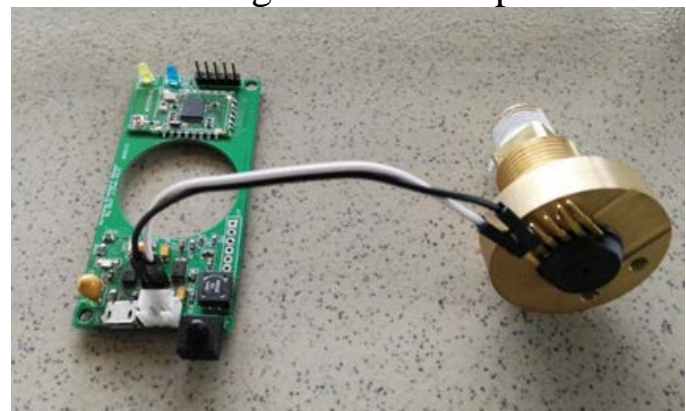

Fig.3. The object of ZigBee wireless pressure sensor 


\section{Sensor Module}

Sensor module uses Freescale Semiconductor's MPX5700 pressure sensor based on the silicon piezo-resistive principle and the sensor has temperature compensation circuits inside, which can measure from 0 to $700 \mathrm{kpa}$ pressure data and the maximum error is only $2.5 \%$ between 0 and $85{ }^{\circ} \mathrm{C}$. The sensor is suitable for the systems based on micro control unit, the working voltage is from $4.75 \mathrm{~V}$ to $5.25 \mathrm{~V}$, and analog output voltage ranges from $0.2 \mathrm{~V}$ to $4.7 \mathrm{~V}$. The air pressure sensor consists of a sensitive element, film temperature compensation and gain level 1, gain level 2 and ground reference conversion circuit, of which sensor circuit design is shown in Figure 4.

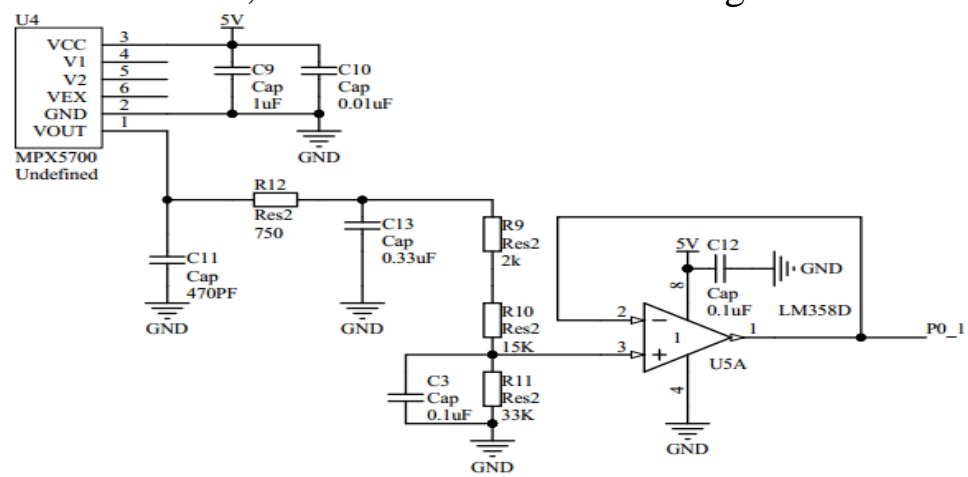

Fig.4. Pressure sensor circuit

\section{The circuit design of charging module}

The microUSB interface of the sensor terminal can be realized to charge the lithium battery. Charging management module is TI BQ24072 chip belonging to linear charging chip, which can set the charging current and time, independently provides power for the system and rechargeable batteries at the same time, possesses over-voltage protection function, and can set maximum input current 1.5A. Only when the dynamic power management (DPPM) channel of chips at the input terminal detects the correct power supply, the charging mode starts, otherwise the charging will stop [6][7].

The charging of chips is divided into three stages. The first stage is adjustment. At this stage, the chip will automatically detects the present battery's voltage. When the voltage is lower than the $\mathrm{V}_{\text {lowv }}$ set by chips, chips will adopt a constant and lower charging current for charging battery in advance; while higher than $\mathrm{V}_{\text {lowv }}$, it will enter into the stage of constant current charge. The second stage is constant current. At this stage, the chips will charge batteries quickly in accordance with the set charging current. During this process, the battery voltage increases rapidly. Until it reaches the rated working voltage of battery, the chip enters the mode of constant voltage charging. The third stage is constant pressure. At this stage, the battery voltage has reached the rated voltage, so the chip will significantly reduce the charging current. Until it reaches the charging time set in advance or detects pin CHG as the high impedance state, the charging will end up. The specific charging process is shown in Figure 5.

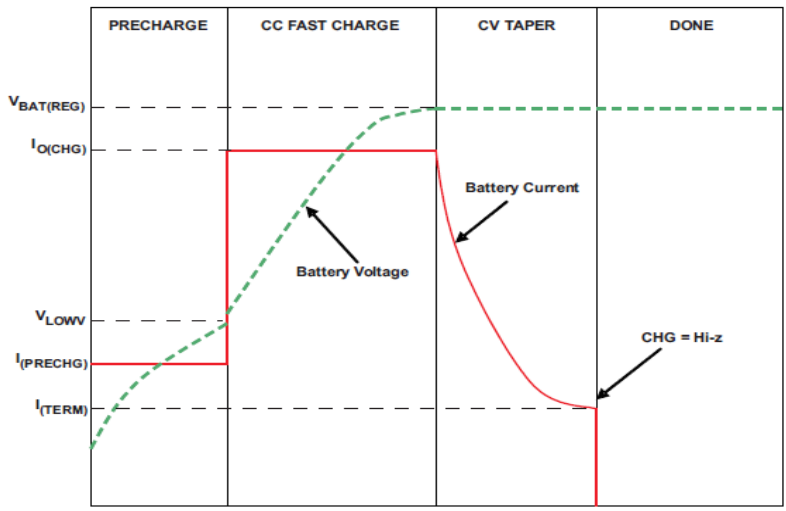

Fig.5. The three stages of BQ24072chip’s charging 
In order to prevent chips overheating during the process of charging, the system will automatically reduce the charging current once detecting chip's temperature is too high no matter at any stage during the process of charging, thus promoting the security during charging. The PGOOD and CHG ports can be respectively connected with a light emitting diode which is used to indicate whether the current state of the external power supply and the charging is completed. The typical connection of chips is shown in Figure 6, and the charging current is set as $800 \mathrm{~mA}$.

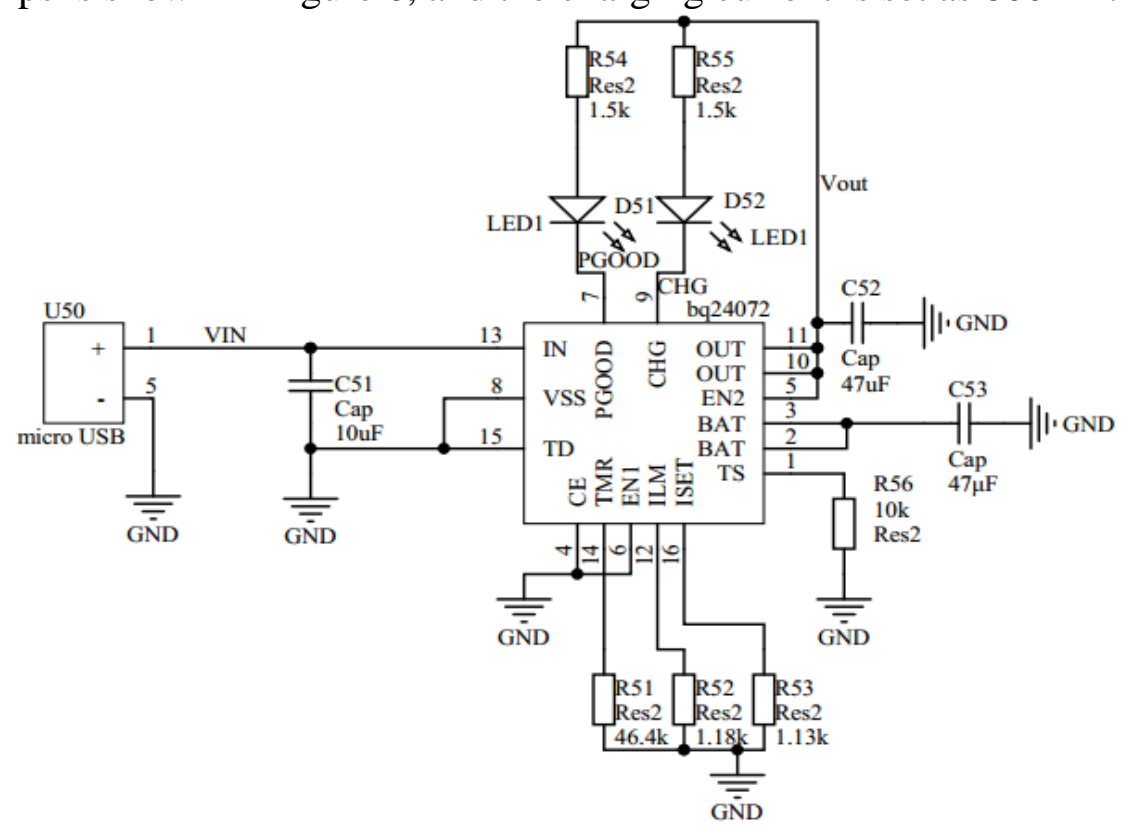

Fig.6. the circuit connection about BQ24072 chip

\section{Wireless transmission module and power step-up, step-down converter circuit}

Wireless transmission module is composed of the system chip CC2530F256 and the peripheral circuit of the ZigBee chip. FAN4855, the power chip to lift and stable pressure, adopted in the circuit design, has lifted the working voltage of lithium battery from $3.7 \mathrm{v}$ to $5 \mathrm{v}$, thus satisfying the operating voltage of pressure sensor. At the same time, the REG1117-3.3 power reducing chip adopted provides the cc2530 wireless chip with 3.3v working voltage.

\section{Design of ZigBee Wireless Pressure Sensor Software}

Software design, based on the TI’s Z-stack protocol stack, supports ZigBee Pro 2007 feature set. The choosing mesh network topology has realized multipath data transmission and improved the reliability and stability of data transmission. In order to reduce system power consumption during idle time and prolong battery life, the design also added the sleep mode.

\section{Conclusion}

The lithium battery technology added in the design of a new generation of air pressure sensor terminal realized the function of terminal node online charging, thus realizing the continuous work of sensors and further meeting practical engineering requirements. A large number of experiments have proved that the sensor is reliable. Moreover, the operation is stable and simple, and it has a broad application prospect and development space.

\section{Acknowledgement}

This work was financially supported by Guangdong Provincial Science and Technology Plan Projects (2014A010103027 and 2016A010101025). 


\section{Reference}

[1] Qiuwei Zhang, Jian Chen, Shuquan Wu. Marine airbags pressure acquisition system based on ZigBee [J]. Journal of Guangdong university of Technology, 2014 31(01)71-73.

[2] Shuquan Wu, Jian Chen, Qiu-wei Zhang, Guoquan Liu. The air pressure monitoring system of airbag group based on C\# [J]. Journal of Guangdong university of Technology, 2014 31(02) 109-112.

[3] Yongfeng Zhong, Yongjun Liu. ZigBee Wireless Sensor Network [M]. Beijing: Beijing university of posts and telecommunications press, 2011.28-30.

[4] Shouwei Gao, Canyang Wu, and etc. ZigBee Technological and Practical Course [M]. Beijing: Beijing University of aeronautics and astronautics press, 2009.

[5] Wangzhi Qiu, Peng Hao, Rob J.Evans. An Efficient Self-healing Process for ZigBee Sensor Networks. IEEE International Symposium on Communications and Information Technologies, ISCIT '07. 2007: 1389 - 1394.

[6] Hongwei Zhang. The design of the principle of lithium ion battery and charging management. Texas Instruments, 2004, 11: 1-15.

[7] V. Potanin, V. Y. Potanin. Li-Ion Battery Charger with Three-Parameter Regulation Loop. IEEE 36th Power Electronics Specialists Conference, 2005: 2836 - 2840. 